\title{
Chaos Synchronization of Two Chaotic Nonlinear Gyros Using Backstepping Design
}

\author{
Rostand M. Davy Loembe-Souamy, Guo-Ping Jiang, Chun-Xia Fan, and Xin-Wei Wang \\ School of Automation, Nanjing University of Posts and Telecommunications, Nanjing 210003, China \\ Correspondence should be addressed to Rostand M. Davy Loembe-Souamy; souamy@163.com and \\ Guo-Ping Jiang; jianggp@njupt.edu.cn
}

Received 28 September 2015; Revised 17 November 2015; Accepted 26 November 2015

Academic Editor: Jonathan N. Blakely

Copyright (C) 2015 Rostand M. Davy Loembe-Souamy et al. This is an open access article distributed under the Creative Commons Attribution License, which permits unrestricted use, distribution, and reproduction in any medium, provided the original work is properly cited.

\begin{abstract}
We investigate the chaos synchronization of two certain chaotic gyros using the backstepping approach. We design a simple controller and verify the stability of the error system by using proper Lyapunov functions. The designed control laws ensure stable controlled and synchronized states for two chaotic nonlinear gyros. Numerical simulations are implemented for illustration and verification of the effectiveness of the backstepping technique.
\end{abstract}

\section{Introduction}

Control and synchronization of chaos as important parts of dynamic systems have great significance to make a good grasp of the fundamentals and basic tools of the science of chaotic dynamics. Synchronization of chaotic systems has become one of the most interesting subjects in chaos theory. Researching chaotic dynamics is the trend of developing technology, since Pecora and Carroll first demonstrated the synchronization of two identical chaotic systems under different initial conditions [1].

The chaos synchronization problem can be defined as two coupled systems conducting coupling evolution in time with given different conditions. In other words, the purpose of synchronization is to use the output of master system to control the slave system, so that the output of slave system achieves asymptotic synchronization with that of master system. Since the pioneering work of synchronization of chaotic systems by Pecora and Caroll, various schemes of synchronization such as backstepping design [2], active control [3], and adaptive control [4] have been successfully employed for chaos synchronization. $H_{\infty}$ synchronization of general chaotic systems with external disturbance was discussed in [5]. The feedback controller was designed to not only guarantee the asymptotic synchronization of master and slave systems but also control the effect of disturbance to an $H_{\infty}$ norm constraint. Additionally, considering the channel noise and parameter mismatch, the technique of robust chaotic $H_{\infty}$ synchronization for Lur'e systems was applied to the secure communication [6]. In $[7,8]$, the passive control technique was applied to chaos synchronization and control.

Gyros are a particularly interesting form of nonlinear system and have attributes of great utility to navigational, aeronautical, and space engineering [9], which have been widely used to evaluate synchronization schemes of chaotic systems. Lei et al. [10] extended the findings of Chen [9] and employed active control method to synchronize two identical chaotic gyros with nonlinear damping. Considering the use of two controllers, however, the active control scheme increases the implementation cost and complexity. Yau [11] presented a robust fuzzy sliding mode control scheme for the synchronization of two chaotic nonlinear gyros subject to uncertainties and external disturbances. Moreover, a growing body of researches [12-17] has emerged in order to address the synchronization of two chaotic nonlinear gyros.

In recent years, the backstepping method [17] of chaos synchronization has attracted many researchers' attention because of its advantages. For example, it performs well on the applicability to a variety of chaotic systems whether they contain external excitation or not, it requires only one controller 
to realize synchronization between chaotic systems, and there are no derivatives in the controller [2]. Backstepping design is a kind of synthetic technique to the controller, which recursively interlaces the choice of a Lyapunov function with the design of feedback control.

In order to reduce the complexity of synchronization schemes and increase the effectiveness and feasibility of control technique, we adopt the backstepping design technique which uses only one controller without three order terms, to realize the synchronization of two chaotic nonlinear gyros. Thus, the main work in this paper is to apply the backstepping technique that designs a simple controller to the synchronization of two certain chaotic nonlinear gyros. In addition, the superiority of the backstepping design is supported by the theoretical analysis and simulations results.

The rest of the paper is organized as follows. In Section 2, a brief description of the gyro system is introduced. In Section 3, we discuss the design of the backstepping controller and verify the stability of error system by using Lyapunov function. In Section 4, numerical simulations are given for illustration and verification of the effectiveness of the backstepping technique. The conclusion is presented in Section 5 .

\section{System Description}

The symmetric gyroscope mounted on a vibrating base is shown in Figure 1. According to Chen [9], the dynamics of a symmetrical gyro with linear-plus-cubic damping of angle $\theta$ can be expressed as

$$
\begin{aligned}
\ddot{\theta} & +\alpha^{2} \frac{(1-\cos \theta)^{2}}{\sin ^{3} \theta}-\beta \sin \theta+c_{1} \dot{\theta}+c_{2} \dot{\theta}^{3} \\
& =f \sin \omega t \sin \theta,
\end{aligned}
$$

where $f \sin \omega t$ represents a parametric excitation, $c_{1} \dot{\theta}$ and $c_{2} \dot{\theta}^{3}$ are linear and nonlinear damping terms, respectively, and $\alpha^{2}\left((1-\cos \theta)^{2} / \sin ^{3} \theta\right)-\beta \sin \theta$ is a nonlinear term.

Given $g(\theta)=-\alpha^{2}\left((1-\cos \theta)^{2} / \sin ^{3} \theta\right)-\beta \sin \theta$ and the states $x_{1}=\theta, x_{2}=\dot{\theta}$, system (1) can be transformed into the following nominal form:

$$
\begin{aligned}
& \dot{x}_{1}=x_{2}, \\
& \dot{x}_{2}=g\left(x_{1}\right)-c_{1} x_{2}-c_{2} x_{2}^{3}+(\beta+f \sin \omega t) \sin \left(x_{1}\right) .
\end{aligned}
$$

The complex dynamics of (2) has been studied by Chen [9] for the value of $f$ in the range of $32<f<36$ and constant values of $\alpha^{2}=100, \beta=1, c_{1}=0.5, c_{2}=0.05$, and $\omega=2$. Figures 2, 3, and 4 illustrate the irregular motion exhibited by system (2) for $f=35.5$ and initial conditions of $\left(x_{1}, x_{2}\right)=(1,-1)$. Figure 5 reveals that the corresponding maximum Lyapunov exponent has a positive value, and thus it can be inferred that the gyro trajectory is in a state of chaotic motion.

Assumption 1. Suppose that there is a constant $l>0$, and then $\left\|g\left(x_{1}\right)-g\left(y_{1}\right)\right\| \leq l\left\|x_{1}-y_{1}\right\|$ holds.

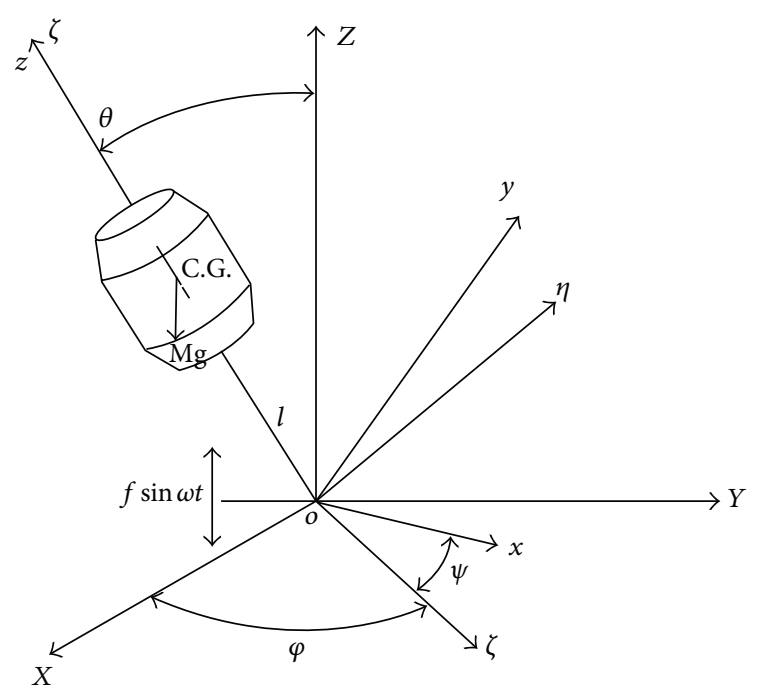

FIGURE 1: A schematic diagram of a symmetric gyroscope [9].

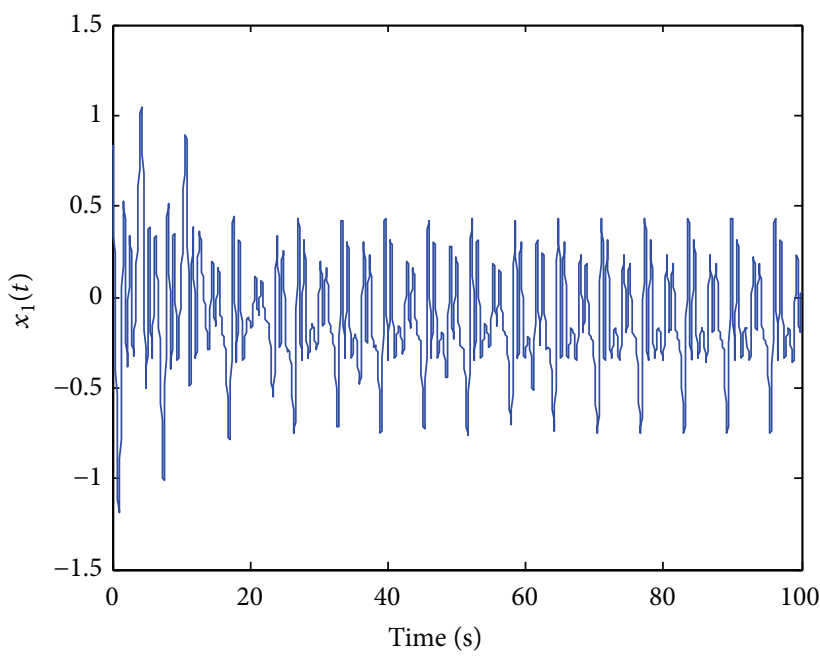

FIgURE 2: Time history of chaotic gyro: $x_{1}$ versus time $t$.

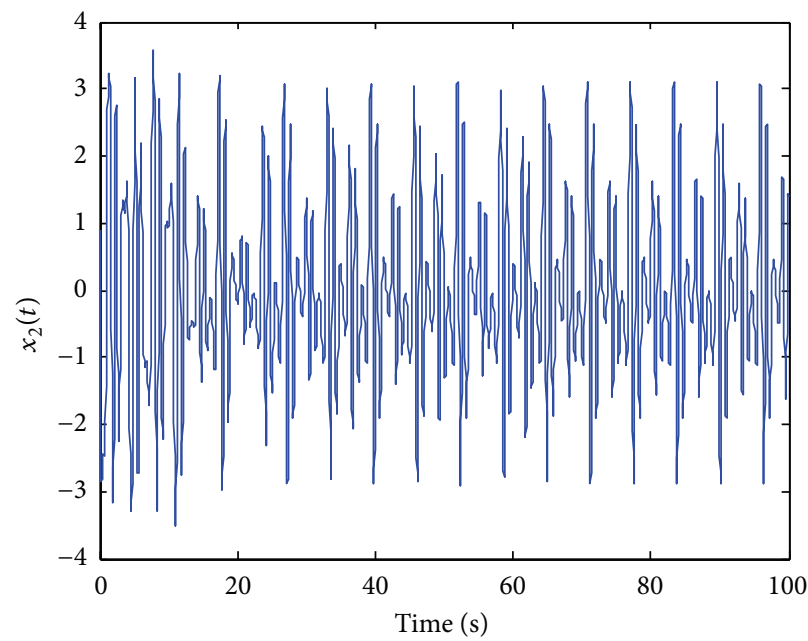

FIgURE 3: Time history of chaotic gyro: $x_{2}$ versus time $t$. 


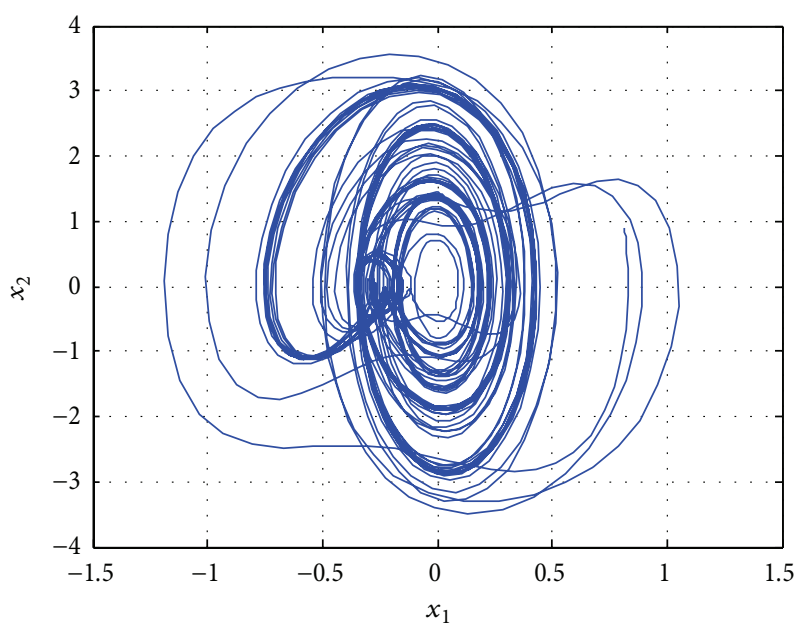

FIGURE 4: Phase plane trajectory of chaotic gyro, $x_{1}$ versus $x_{2}$.

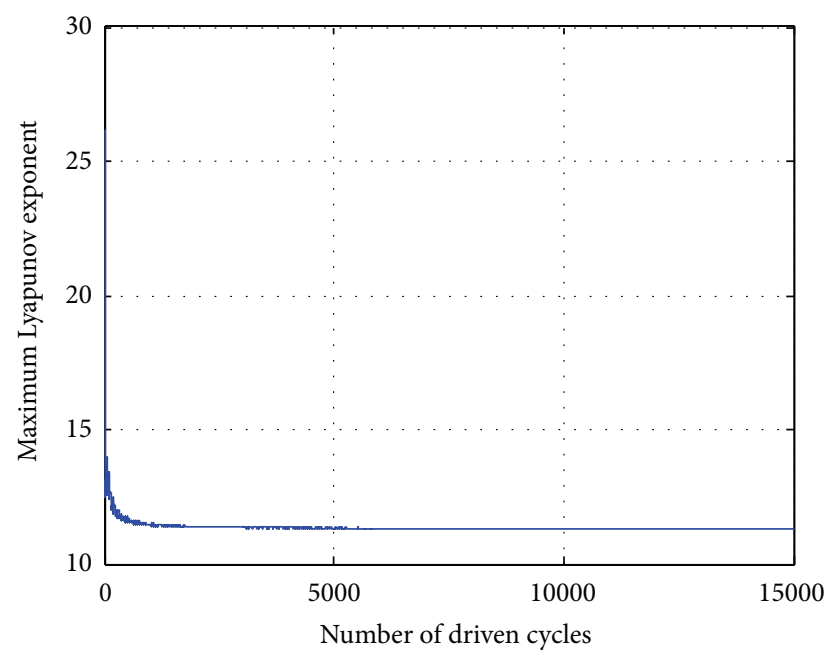

Figure 5: Maximum Lyapunov exponent of gyro trajectory plotted as function of number of drive cycles.

We take gyroscope (2) as the master system. By using the backstepping technique, we design controller $u(t)$ in the following slave system:

$$
\begin{aligned}
\dot{y}_{1}= & y_{2}, \\
\dot{y}_{2}= & g\left(y_{1}\right)-c_{1} y_{2}-c_{2} y_{2}^{3}+(\beta+f \sin \omega t) \sin \left(y_{1}\right) \\
& -u(t),
\end{aligned}
$$

where $u(t)$ is an appropriate control signal.

The goal of the current control problem is to design $u(t)$, so that, for any initial conditions of two systems (2) and (3), the behavior of the slave system converges to that of the master system; that is,

$$
\lim _{t \rightarrow \infty}\|x(t)-y(t)\| \longrightarrow 0
$$

where $\|\cdot\|$ is the Euclidean norm of a vector.
We define the error states between the master system and slave system as

$$
\begin{aligned}
& e_{1}=x_{1}-y_{1}, \\
& e_{2}=x_{2}-y_{2} .
\end{aligned}
$$

Dynamics equation (6) of these errors can be obtained directly by subtracting (3) from (2):

$$
\begin{aligned}
\dot{e}_{1}= & e_{2}, \\
\dot{e}_{2}= & -c_{1} e_{2}+g\left(x_{1}\right)-g\left(y_{1}\right)-c_{2}\left(x_{2}^{3}-y_{2}^{3}\right) \\
& +(\beta+f \sin \omega t)\left(\sin x_{1}-\sin y_{1}\right)+u(t) \\
= & -c_{1} e_{2}+g\left(x_{1}\right)-g\left(x_{1}-e_{1}\right)-c_{2} x_{2}^{3} \\
& +c_{2}\left(x_{2}-e_{2}\right)^{3} \\
& +(\beta+f \sin \omega t)\left(\sin \left(x_{1}\right)-\sin \left(x_{1}-e_{1}\right)\right) \\
& +u(t) .
\end{aligned}
$$

\section{Backstepping Design}

Compared with other controllers, we design simple controller $u(t)$ without three order terms.

Based on the backstepping method, error variable $\omega_{2}$ needs to be defined: $\omega_{2}=e_{2}-\alpha_{1}\left(e_{1}\right)$, where $\alpha_{1}\left(e_{1}\right)=-e_{1}$, and then we get $\omega_{2}=e_{2}+e_{1}$.

When $\omega_{2}=e_{2}+e_{1}$, error dynamics equation (6) is depicted as

$$
\begin{aligned}
\dot{e}_{1}= & \omega_{2}-e_{1}, \\
\dot{e}_{2}= & -c_{1} e_{2}+g\left(x_{1}\right)-g\left(x_{1}-e_{1}\right)-c_{2} x_{2}^{3}+c_{2}\left(x_{2}-e_{2}\right)^{3} \\
& +(\beta+f \sin \omega t)\left(\sin \left(x_{1}\right)-\sin \left(x_{1}-e_{1}\right)\right) \\
& +u(t) .
\end{aligned}
$$

Considering $\left(e_{1}, \omega_{2}\right)$ subspace given by

$$
\begin{aligned}
\dot{e}_{1}= & \omega_{2}-e_{1} \\
\dot{\omega}_{2}= & e_{2}-c_{1} e_{2}+g\left(x_{1}\right)-g\left(x_{1}-e_{1}\right)-c_{2} x_{2}^{3} \\
& +(\beta+f \sin \omega t)\left(\sin \left(x_{1}\right)-\sin \left(x_{1}-e_{1}\right)\right) \\
& +c_{2}\left(x_{2}-e_{2}\right)^{3}+u(t)
\end{aligned}
$$

we design controller $u(t)$ as follows:

$$
\begin{aligned}
u(t)= & c_{1} e_{2}-e_{2} \\
& -(\beta+f \sin \omega t)\left(\sin \left(x_{1}\right)-\sin \left(x_{1}-e_{1}\right)\right) \\
& -\frac{1}{\varepsilon_{1}} l^{2} \omega_{2}^{2}-\frac{c_{2}}{\varepsilon_{2}} \delta^{2} \omega_{2}^{2}-c_{2} \delta \omega_{2}^{2}-\frac{e_{1} e_{2}}{\omega_{2}},
\end{aligned}
$$




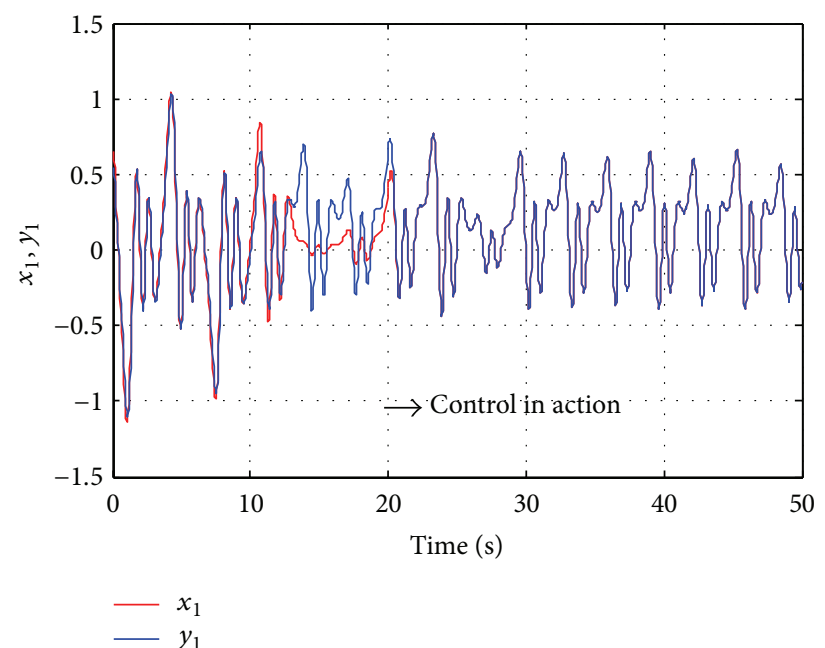

(a)

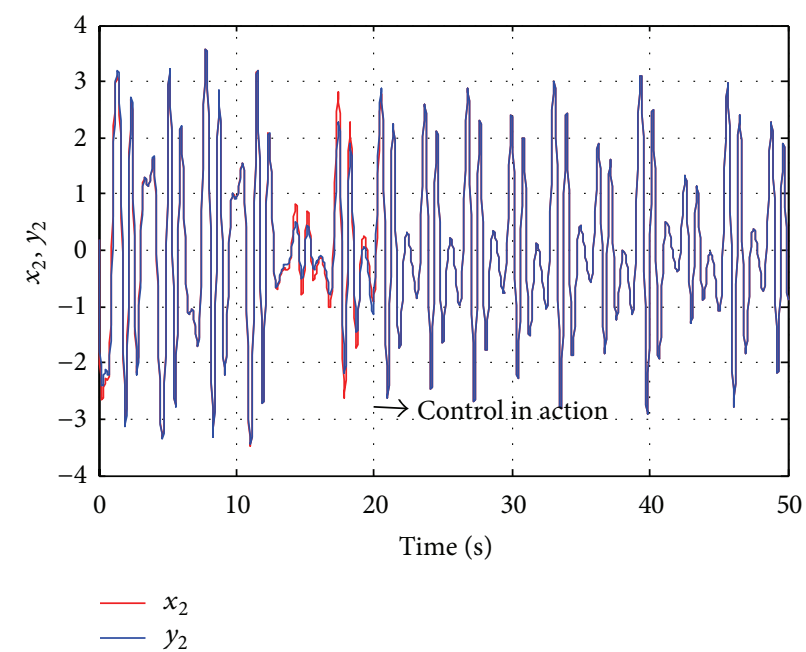

(b)

FIGURE 6: Time responses of controlled chaotic gyro system: master and slave system outputs are $x_{1}, x_{2}$ (red) and $y_{1}, y_{2}$ (blue), respectively. Note that the control $u(t)$ is activated at $t=20 \mathrm{~s}$.

where $\varepsilon_{1}>0, \varepsilon_{2}>0, \varepsilon_{1}+\varepsilon_{2} \mathcal{c}_{2}<1$, and $\varepsilon_{1}$ and $\varepsilon_{2}$ are constant parameter signals, which are used to adjust the controller in the slave system. From (9), we can see that, by means of the backstepping algorithm for strict feedback nonlinear continuous systems, the controller is easy to realize.

Remark 2. In [17], the authors proposed a controller with three order terms for chaos synchronization in gyros systems, while we have already removed these terms in our controller (see (9)), so that the complexity is reduced.

Theorem 3. Consider the master-slave system given in (2) and (3). The two systems can be globally asymptotically synchronized by control $u(t)$ defined in (9). That is, error dynamical system (6) is globally exponentially stable about the origin.

Proof. Choose the Lyapunov function:

$$
V_{2}\left(e_{1}, \omega_{2}\right)=V_{1}\left(e_{1}\right)+\frac{1}{2} \omega_{2}^{2}
$$

where $V_{1}\left(e_{1}\right)=(1 / 2) e_{1}^{2}$.

The derivative of (10) is

$$
\begin{aligned}
\dot{V}_{2} & =e_{1} \dot{e}_{1}+\omega_{2} \dot{\omega}_{2}=-e_{1}^{2}+\omega_{2}\left[-c_{1} e_{2}+g\left(x_{1}\right)-g\left(y_{1}\right)\right. \\
& -c_{2} x_{2}^{3}+c_{2} y_{2}^{3}+(\beta+f \sin \omega t)\left(\sin \left(x_{1}\right)-\sin \left(y_{1}\right)\right) \\
& \left.-e_{1}+u(t)\right]=-e_{1}^{2}+\omega_{2}\left[g\left(x_{1}\right)-g\left(y_{1}\right)\right. \\
& \left.-c_{2}\left(x_{2}^{3}-y_{2}^{3}\right)-\frac{1}{\varepsilon_{1}} l^{2} \omega_{2}^{2}-\frac{c_{2}}{\varepsilon_{2}} \delta^{2} \omega_{2}^{2}-c_{2} \delta \omega_{2}^{2}\right] .
\end{aligned}
$$

From Assumption 1, we obtain

$$
\begin{aligned}
& \omega_{2}\left(g\left(x_{1}\right)-g\left(x_{1}-e_{1}\right)\right) \leq l\left\|\omega_{2}\right\|\left\|e_{1}\right\| \\
& \quad \leq \frac{1}{\varepsilon_{1}} l^{2}\left\|\omega_{2}\right\|^{2}+\varepsilon_{1}\left\|e_{1}\right\|^{2}=\frac{1}{\varepsilon_{1}} l^{2} \omega_{2}^{2}+\varepsilon_{1} e_{1}^{2} .
\end{aligned}
$$

Because $x_{2}$ and $y_{2}$ are bounded, there is a constant $\delta>0$ such that $\left\|y_{2}^{3}-x_{2}^{3}\right\| \leq \delta\left\|y_{2}-x_{2}\right\|$, and we get

$$
\begin{aligned}
\omega_{2}\left(c_{2} y_{2}^{3}-c_{2} x_{2}^{3}\right) \leq & \left\|\omega_{2}\right\| c_{2} \delta\left\|y_{2}-x_{2}\right\| \leq\left\|\omega_{2}\right\| c_{2} \delta\left\|e_{2}\right\| \\
= & \left\|\omega_{2}\right\| c_{2} \delta\left\|\omega_{2}-e_{1}\right\| \\
\leq & \left\|\omega_{2}\right\| c_{2} \delta\left(\left\|\omega_{2}\right\|+\left\|e_{1}\right\|\right) \\
= & c_{2} \delta\left\|\omega_{2}\right\|^{2}+c_{2} \delta\left\|\omega_{2}\right\|\left\|e_{1}\right\| \\
\leq & c_{2} \delta\left\|\omega_{2}\right\|^{2}+\frac{\delta^{2}}{\varepsilon_{2}} c_{2}\left\|\omega_{2}\right\|^{2} \\
& +\varepsilon_{2} c_{2}\left\|e_{1}\right\|^{2} \\
= & c_{2} \delta \omega_{2}^{2}+\frac{c_{2}}{\varepsilon_{2}} \delta^{2} \omega_{2}^{2}+\varepsilon_{2} c_{2} e_{1}^{2} .
\end{aligned}
$$

According to analysis above, we get

$$
\begin{aligned}
\dot{V} \leq & -e_{1}^{2}+\frac{1}{\varepsilon_{1}} l^{2} \omega_{2}^{2}+\varepsilon_{1} e_{1}^{2}+c_{2} \delta \omega_{2}^{2}+\frac{c_{2}}{\varepsilon_{2}} \delta^{2} \omega_{2}^{2}+\varepsilon_{2} c_{2} e_{1}^{2} \\
& -\frac{1}{\varepsilon_{1}} l^{2} \omega_{2}^{2}-c_{2} \delta \omega_{2}^{2}-\frac{c_{2}}{\varepsilon_{2}} \delta^{2} \omega_{2}^{2} \\
= & -e_{1}^{2}+\varepsilon_{1} e_{1}^{2}+\varepsilon_{2} c_{2} e_{1}^{2},
\end{aligned}
$$

where $\varepsilon_{1}+\varepsilon_{2} \mathcal{C}_{2}<1$, and then $\dot{V}<0$. Error dynamical system (6) will converge to zero as $t \rightarrow \infty$, while equilibrium $(0,0)$ remains globally asymptotically stable. In the above analysis, $\dot{V}<0$ is bounded away from zero for all points except where $e_{1}=0, e_{2}=0$. Therefore, the error of synchronization is verified to be asymptotically stable. 


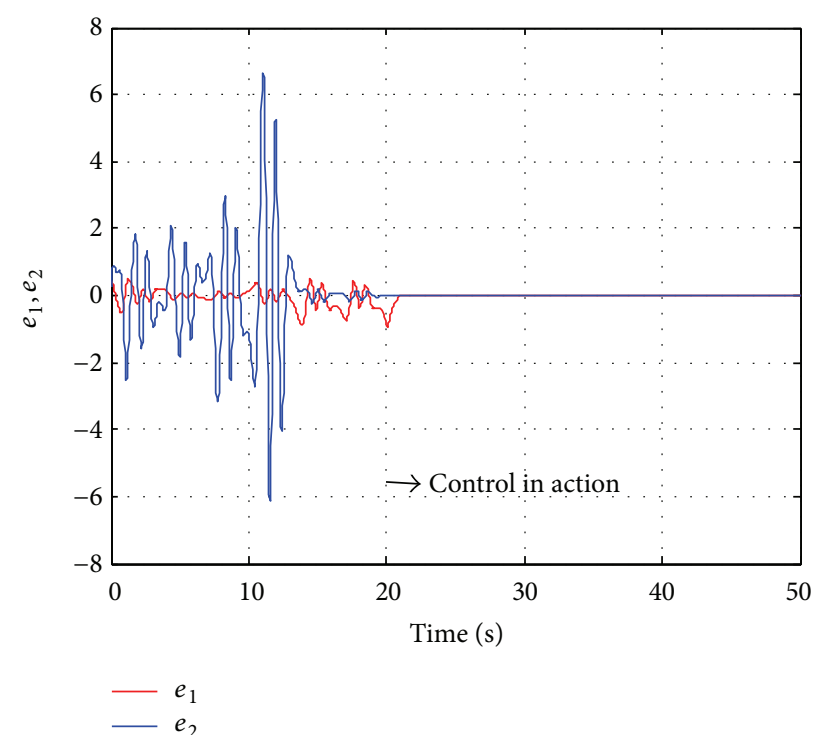

FIgURE 7: Time response of error states. Note that control $u(t)$ is activated at $t=20 \mathrm{~s}$.

\section{Simulations Results}

The parameters of the nonlinear gyros systems are specified as follows: $\alpha^{2}=100, \beta=1, c_{1}=0.5, c_{2}=0.05$, and $f=35.5$, which is shown in Section 2 and gives rise to a chaotic state. The initial conditions are defined as $x_{1}(0)=1, x_{2}(0)=-1$, $y_{1}(0)=1.6$, and $y_{2}(0)=0.8$. In the proposed design, the controller is determined in accordance with the backstepping control law.

Figure 6 shows the time responses of controlled chaotic gyro synchronization system: master system and slave system outputs are $x_{1}, x_{2}$ (red) and $y_{1}, y_{2}$ (blue), respectively, where the control signal is activated at $t=20 \mathrm{~s}$. This result demonstrates that the output of slave system (3) achieves asymptotic synchronization with the output of master system (2).

Error states of coupled system are defined as $e_{1}=x_{1}-y_{1}$ and $e_{2}=x_{2}-y_{2}$ in the backstepping controller. Figures 7 and 8 show the time responses of the error states and control signal $u(t)$, respectively. The results show that the error states are regulated to zero asymptotically following activation of the control signal at $t=20 \mathrm{~s}$.

\section{Conclusions}

Based on Lyapunov stability theory, a controller which employed the backstepping approach has been designed for the synchronization of two chaotic nonlinear gyros. The backstepping technique we have applied allows for flexibility in the controller design and global stability based on the appropriate choice of Lyapunov functions. The simulation results show that the synchronization scheme of backstepping approach is effective and has low complexity. Compared with the existing synchronization schemes, our design avoids the complexity of behavior on the chaos controller and therefore has a lower implementation cost.

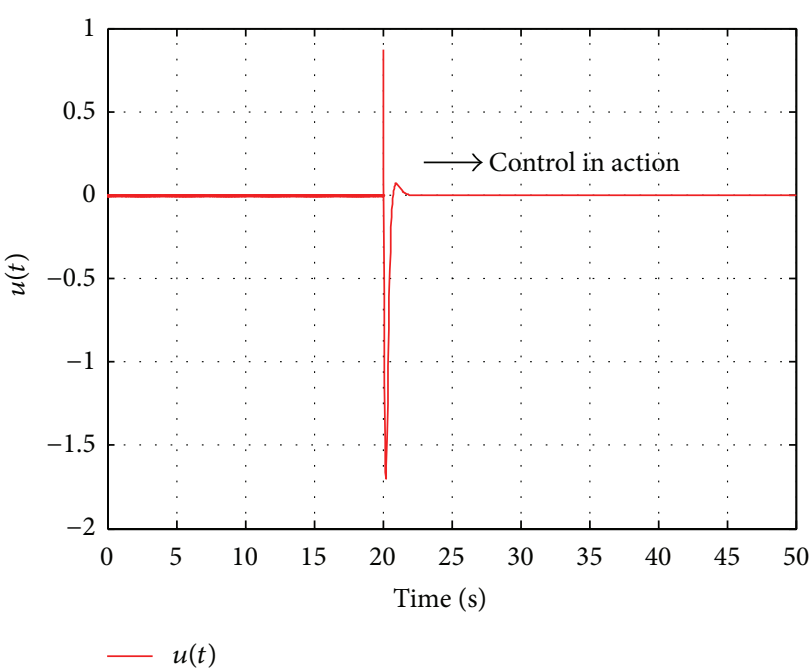

FIgURE 8: Variation of control action over time. Note that control $u(t)$ is activated at $t=20 \mathrm{~s}$.

In the future, we will improve the design of controller with consideration of more realistic situations, such as the uncertainties and disturbance, to realize the synchronization of two uncertain chaotic nonlinear gyros.

\section{Conflict of Interests}

The authors declare that there is no conflict of interests regarding the publication of this paper.

\section{References}

[1] L. M. Pecora and T. L. Carroll, "Synchronization in chaotic systems," Physical Review Letters, vol. 64, no. 8, pp. 821-824, 1990.

[2] X. H. Tan, J. Y. Zhang, and Y. R. Yang, "Synchronizing chaotic systems using backstepping design," Chaos, Solitons \& Fractals, vol. 16, no. 1, pp. 37-45, 2003.

[3] M.-C. Ho and Y.-C. Hung, "Synchronization of two different systems by using generalized active control," Physics Letters A, vol. 301, no. 5-6, pp. 424-428, 2002.

[4] S. H. Chen and J. H. Lü, "Synchronization of an uncertain unified chaotic system via adaptive control," Chaos, Solitons \& Fractals, vol. 14, no. 4, pp. 643-647, 2002.

[5] S. M. Lee, D. H. Ji, J. H. Park, and S. C. Won, " $H_{\infty}$ synchronization of chaotic systems via dynamic feedback approach," Physics Letters A, vol. 372, no. 29, pp. 4905-4912, 2008.

[6] J. A. K. Suykens, P. F. Curran, J. Vandewalle, and L. O. Chua, "Robust nonlinear $\mathrm{H}_{\infty}$ synchronization of chaotic Lur'e systems," IEEE Transactions on Circuits and Systems I: Fundamental Theory and Applications, vol. 44, no. 10, pp. 891-904, 1997.

[7] J. Yao, Z.-H. Guan, and D. J. Hill, "Passivity-based control and synchronization of general complex dynamical networks," Automatica, vol. 45, no. 9, pp. 2107-2113, 2009.

[8] X.-J. Wu, J.-S. Liu, and G.-R. Chen, "Chaos synchronization of Rikitake chaotic attractor using the passive control technique," Nonlinear Dynamics, vol. 53, no. 1-2, pp. 45-53, 2008.

[9] H.-K. Chen, "Chaos and chaos synchronization of a symmetric gyro with linear-plus-cubic damping," Journal of Sound and Vibration, vol. 255, no. 4, pp. 719-740, 2002. 
[10] Y. M. Lei, W. Xu, and H. C. Zheng, "Synchronization of two chaotic nonlinear gyros using active control," Physics Letters A, vol. 343, no. 1-3, pp. 153-158, 2005.

[11] H.-T. Yau, "Chaos synchronization of two uncertain chaotic nonlinear gyros using fuzzy sliding mode control," Mechanical Systems and Signal Processing, vol. 22, no. 2, pp. 408-418, 2008.

[12] N. Vasegh and V. J. Majd, "Adaptive fuzzy synchronization of discrete-time chaotic systems," Chaos, Solitons \& Fractals, vol. 28, no. 4, pp. 1029-1036, 2006.

[13] M. Roopaei, M. Z. Jahromi, and S. Jafari, "Adaptive gain fuzzy sliding mode control for the synchronization of nonlinear chaotic gyros," Chaos, vol. 19, no. 1, Article ID 013125, 2009.

[14] F.-H. Min, "Generalized projective synchronization between two chaotic gyros with nonlinear damping," Chinese Physics $B$, vol. 20, no. 10, Article ID 100503, 2011.

[15] M. P. Aghababa and H. P. Aghababa, "Chaos synchronization of gyroscopes using an adaptive robust finite-time controller," Journal of Mechanical Science and Technology, vol. 27, no. 3, pp. 909-916, 2013.

[16] C.-C. Yang and C.-J. Ou, "Adaptive terminal sliding mode control subject to input nonlinearity for synchronization of chaotic gyros," Communications in Nonlinear Science and Numerical Simulation, vol. 18, no. 3, pp. 682-691, 2013.

[17] B. A. Idowu, U. E. Vincent, and A. N. Njah, "Control and synchronization of chaos in nonlinear gyros via backstepping design," International Journal of Nonlinear Science, vol. 5, no. 1, pp. 11-19, 2008. 


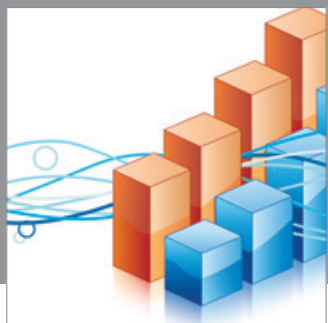

Advances in

Operations Research

mansans

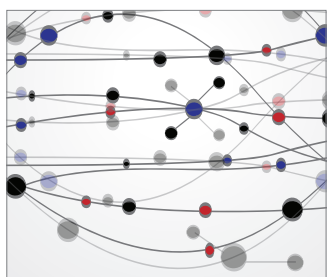

The Scientific World Journal
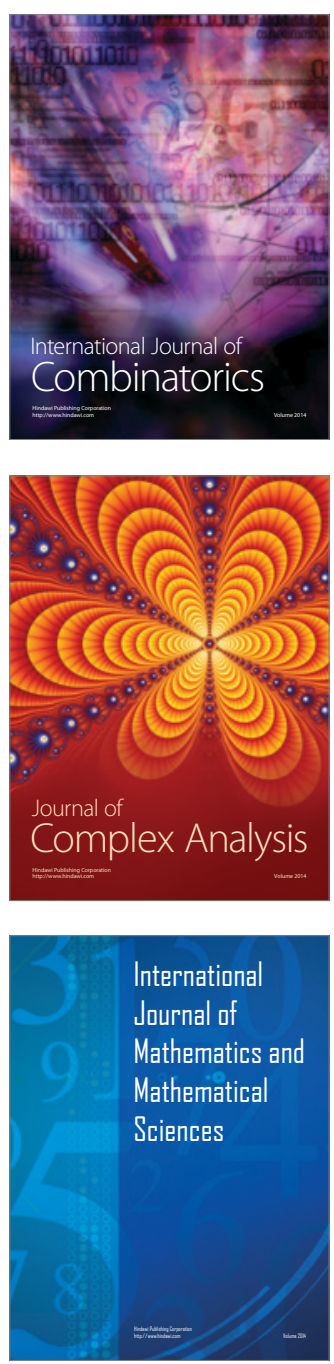
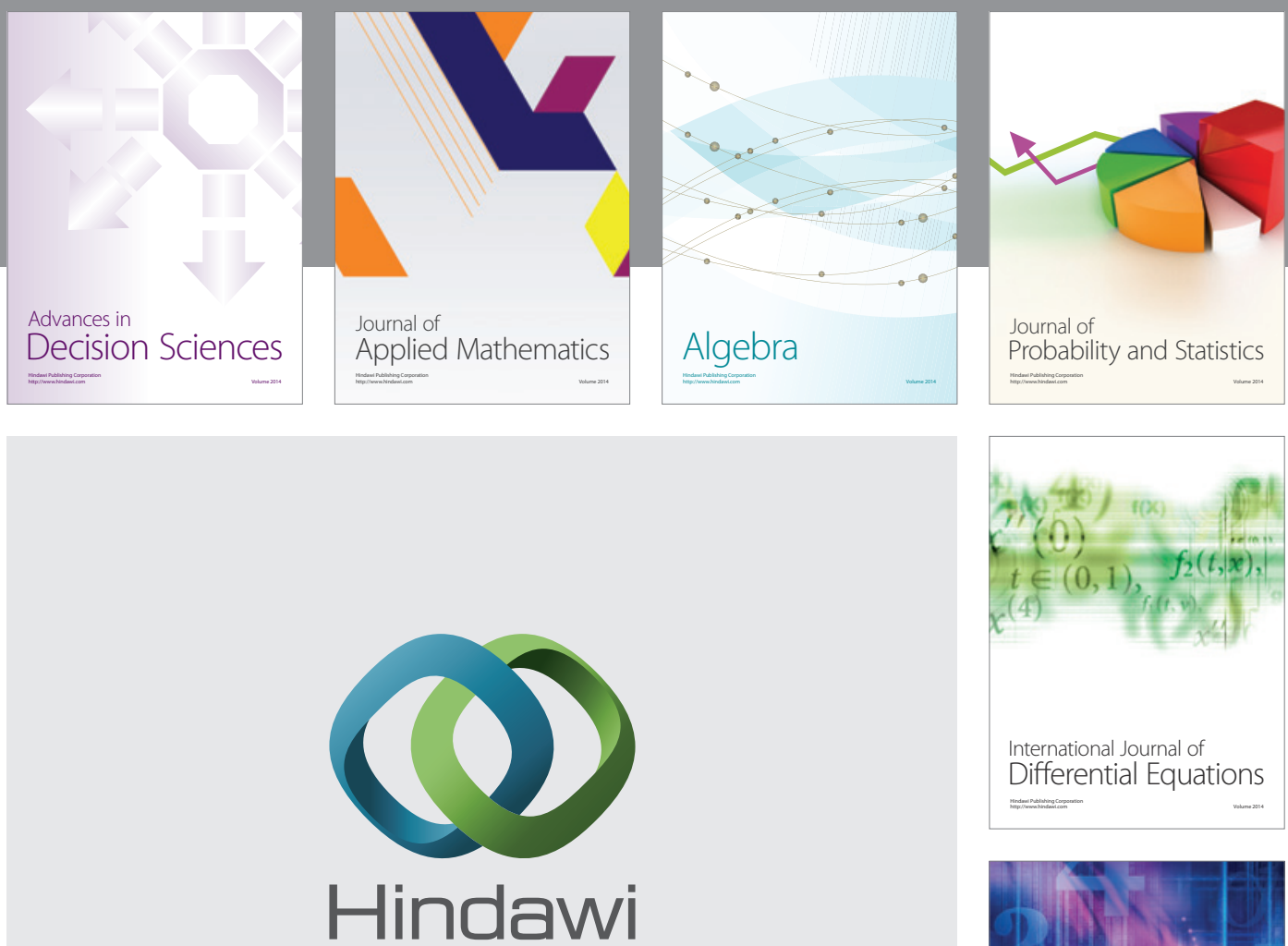

Submit your manuscripts at http://www.hindawi.com
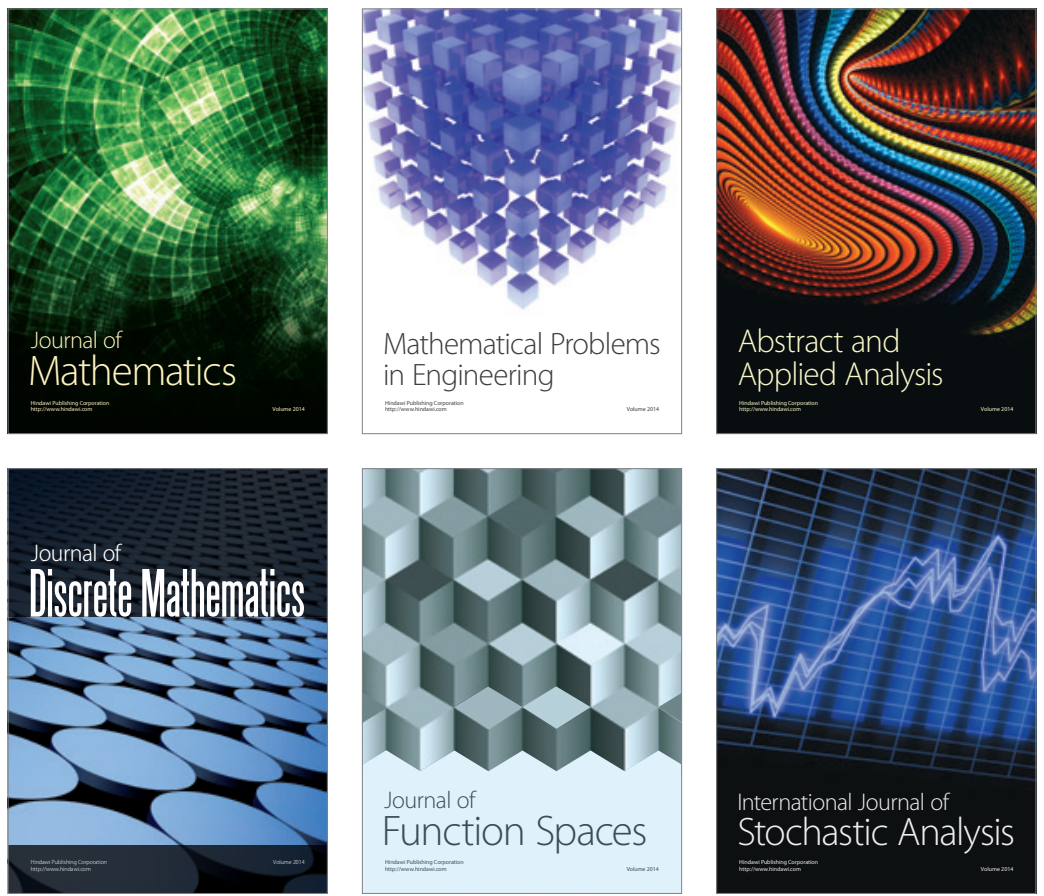

Journal of

Function Spaces

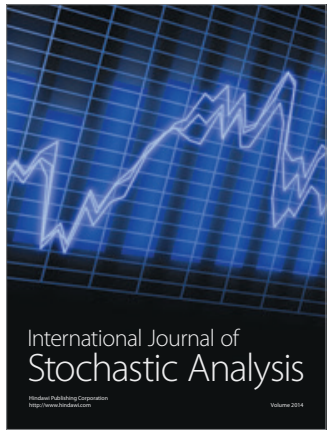

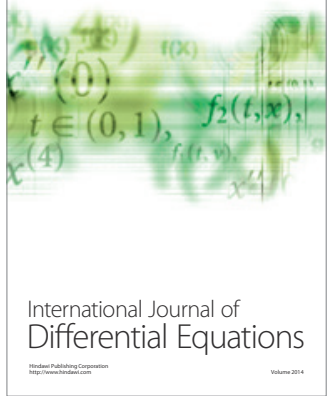
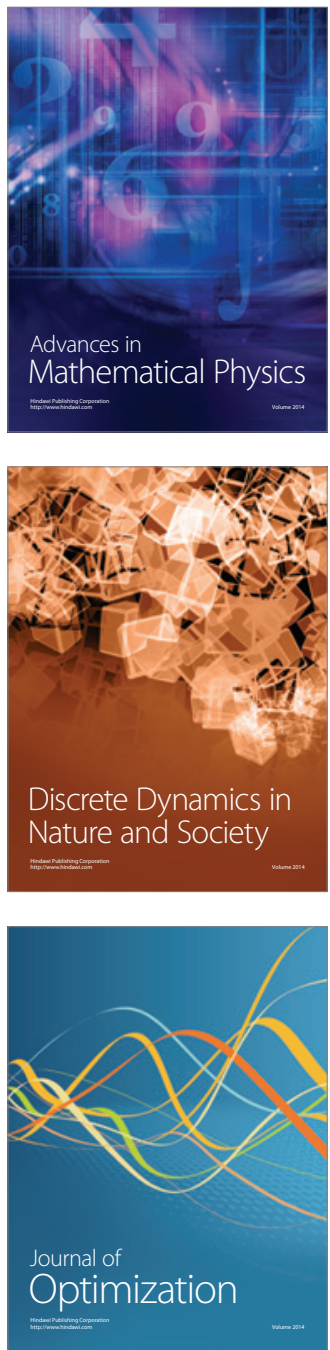